\title{
Building Blue Stragglers with Stellar Collisions
}

\author{
Evert Glebbeek and Onno R. Pols
}

Sterrekundig Instituut Utrecht, P.O. Box 80000, 3508 TA Utrecht, The Netherlands

\begin{abstract}
The evolution of stellar collision products in cluster simulations has usually been modelled using simplified prescriptions. Such prescriptions either replace the collision product with an (evolved) main sequence star, or assume that the collision product was completely mixed during the collision.

It is known from hydrodynamical simulations of stellar collisions that collision products are not completely mixed, however. We have calculated the evolution of stellar collision products and find that they are brighter than normal main sequence stars of the same mass, but not as blue as models that assume that the collision product was fully mixed during the collision.
\end{abstract}

Keywords. Stars: blue stragglers, evolution, formation

\section{Introduction}

The aim of the MODEST collaboration (Hut et al. (2003)) is to model and understand dense stellar systems, which requires a good understanding of what happens when two single stars or binary systems undergo a close encounter. A possible outcome of such an encounter is a collision followed by the merging of two or more stars. This is a possible formation channel for blue straggler stars (e.g. Sills et al. (1997)). Understanding the formation and evolution of blue stragglers is important for understanding the Hertzsprung-Russell diagram of clusters.

\section{Method}

We have developed a version of the Eggleton stellar evolution code (Eggleton (1971), Pols et al. (1995)) that can import the output of a collision calculation and calculate the subsequent evolution of the remnant, in principle without human intervention.

We have used this code to calculate detailed evolution models of collision remnants from the $N$-body simulation of M67 by Hurley et al. (2005) and compared these with normal main sequence stars of the same mass as well as homogeneous models with the same average abundances. The post-merger profiles were calculated with the parametrised code of Lombardi et al. (2002).

The collisions in the $N$-body simulation span a range of total masses $M=M_{1}+$ $M_{2}$, mass ratio $q=M_{2} / M_{1}$ and time of collision $t$. We have also calculated a grid of models spanning four collision times $(t=2800,3100,3400$ and $3700 \mathrm{Myr})$, four mass ratios $(q=0.4,0.6,0.8$ and 1.0$)$ and six total masses $(M=1.5,1.6,1.7,1.8,1.9$ and 2.0$)$, which covers the parameter space of collisions found in the $N$-body simulation. 


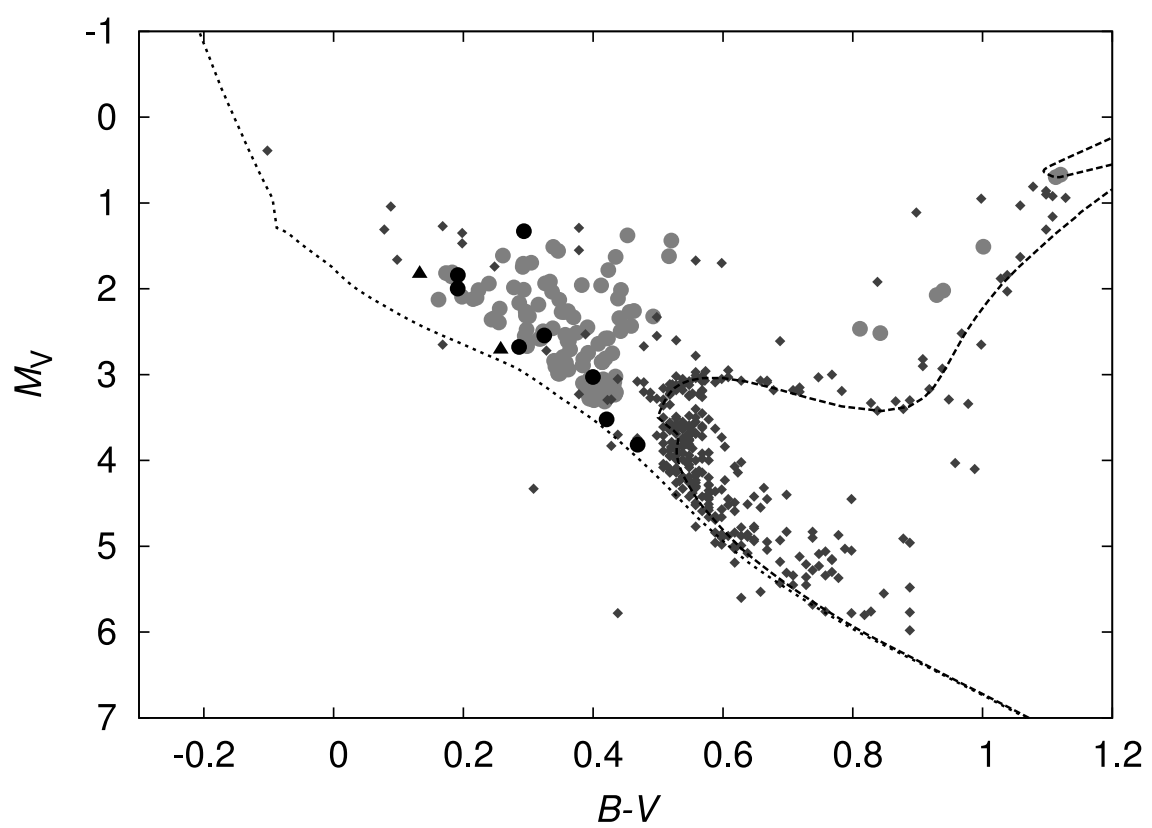

Figure 1. Colour-magnitude diagram of the open cluster M67 $(\downarrow)$. Overplotted are the locations of our models at $4 \mathrm{Gyr}$, the age of M67. The black $(\bullet, \boldsymbol{\Delta})$ symbols are collisions from the M67 simulation. Two of these are double collisions, which are indicated by $\boldsymbol{\Lambda}$. The grey $(\bullet)$ symbols are from our larger grid.

\section{Results and Conclusions}

Compared to normal stars, collision products are helium enhanced. Most of the helium enhancement is in the interior and does not affect the opacity of the envelope. The helium enhancement does increase the mean molecular weight and therefore the luminosity of the star. This decreases the remaining lifetime of collision products compared to normal stars of the same mass and can be important for the predicted number of blue stragglers from cluster simulations. The increased luminosity changes the distribution of blue stragglers in the colour-magnitude diagram, moving it above the extension of the main sequence.

The evolution track of a fully mixed model can be significantly bluer than a selfconsistently calculated evolution track of a merger remnant. Fully mixed models are closer to the zero age main sequence.

Our grid of models covers most of the observed blue straggler region of M67 (Fig. 1). A better coverage of the blue part of the region can be achieved by increasing the upper mass limit in the grid. The brightest observed blue straggler falls outside the region of our grid because it requires at least a double collision to explain.

\section{References}

Eggleton P. P., 1971, MNRAS, 151, 351

Hurley J. R., Pols O. R., Aarseth S. J., \& Tout C. A., 2005, MNRAS, 363, 293

Hut P. et al. 2003, New Astronomy, 8, 337

Lombardi Jr. J. C., Warren J. S., Rasio F. A., Sills A., \& Warren A. R., 2002, ApJ, 568, 939

Pols O. R., Tout C. A., Eggleton P. P., \& Han Z., 1995, MNRAS, 274, 964

Sills A., Lombardi Jr. J. C., Bailyn C. D., Demarque P., Rasio F. A., \& Shapiro S. L., 1997, ApJ, 487, 290 